\title{
Erratum zu: Grundlagen und Wirkungsweise von zielgerichteten Therapien*
}

\author{
Christian F. Singer \\ Arbeitsgruppe Senologie der Klinischen Abteilung für Allgemeine Gynäkologie und Gynäkologische \\ Onkologie, Universitätsklinik für Frauenheilkunde, Medizinische Universität Wien, Wien, Österreich
}

Eingelangt am 18. April 2010, angenommen am 7. Mai 2010, online veröffentlicht am 24. August 2010

\section{Principles and method of action of targeted therapies}

Summary. Conventional cytotoxic therapy is usually characterized by low specificity and considerable side effects. Targeted therapy, by contrast, allows for a specific inhibition with an acceptable side effect profile. A prerequisite for the development of such a therapy, however, is the identification and characterization of the molecular mechanisms that lead to tumor growth. Antibody-based targeted therapies usually attack cell membranebound or extracellular proteins, while tyrosin kinase inhibitors usually act at intracellular domains of transmembranous proteins. Both strategies ultimately lead to an inhibition of the signal transduction cascade and thereby block increased cell proliferation, metastasis, or the production of new blood or lymph vessels.

Key words: Signal transduction, targeted therapy, angiogenesis, tyrosin kinase inhibitor

Zusammenfassung. Während die konventionelle zytostatische Krebstherapie durch eine geringe Spezifität und relativ ausgeprägte Nebenwirkungen charakterisiert ist, erlaubt die spezifische Inhibierung des Tumorwachstums eine zielgerichtete Therapie bei zumeist günstigem Nebenwirkungsprofil. Voraussetzung für eine derartige Therapie ist jedoch die Identifizierung und Charakterisierung der molekularen Mechanismen, die zum Tumorwachstum führen. Zielgerichtete Therapien greifen zumeist als Antikörper an der Zelloberfläche befindliche oder extrazelluläre Proteine an, während Tyrosinkinaseinhibitoren in der Zelle, oft an der intrazellulären Domäne von transmembranären Rezeptoren, binden. Beide Strategien führen letztlich zu einer Hemmung in der Signaltransduktionskaskade und verhindern

${ }^{*}$ Die unter DOI 10.1007/s10354-010-0817-y online first veröffentlichte Fassung ist im nachfolgenden Beitrag ergänzt bzw. berichtigt.

Korrespondenz: Ao. Univ.-Prof. Dr. Christian F. Singer, Arbeitsgruppe Senologie der Klinischen Abteilung für Allgemeine Gynäkologie und Gynäkologische Onkologie, Universitätsklinik für Frauenheilkunde, Medizinische Universität Wien, Währinger Gürtel 18-20, 1090 Wien, Österreich.

Fax: ++43-1-40400 2323, E-Mail: christian.singer@meduniwien.ac.at über diesen Mechanismus vermehrte Zellteilung, Metastasierung oder die Neubildung von Blut- und Lymphgefäßen.

Schlüsselwörter: Signaltransduktion, zielgerichtete Therapie, Angiogenese, Tyrosinkinase-Hemmer

\section{Inhibition der Signaltransduktion}

\section{HER2-Signaltransduktionshemmung als therapeutisches Ziel}

Der Epidermal Growth Factor Receptor (EGFR) bildet gemeinsam mit den strukturell verwandten Rezeptoren HER2, HER3 und HER4 eine Familie von membranständigen Tyrosinkinaserezeptoren, die bei der Regulation von Zellproliferation und biologischem Verhalten eine große Bedeutung haben. Durch die hochspezifische Bindung eines Liganden kommt es zur Entstehung von Rezeptordimeren, was in der Regel mit einer Auto-und Transphosphorylierung der intrazellulären Rezeptordomäne und mit einer Rezeptoraktivierung verbunden ist (Abb. 1). Notable Ausnahmen von dieser Regel sind HER2, für den bislang noch kein Ligand beschrieben wurde, und HER3, der keine intrinsische Tyrosinkinaseaktivität besitzt.

Damit scheint die biologische Rolle von HER2 bei der Signaltransduktionsaktivierung primär darin zu bestehen, dass er als Dimerisierungspartner für andere Mitglieder der HER-Familie dient. Die Aktivierung der Signaltransduktion durch HER1-4 ist ein hochkomplexer und in der gesunden Zelle exakt gesteuerter Prozess, der auf Störungen in der Rezeptorexpression bzw. -aktivierung besonders empfindlich ist. So führt die in etwa $20 \%$ aller Mammakarzinome nachweisbare Überexpression von HER2 als Folge einer Amplifikation des HER2-Locus in Tumorzellen zu einer konstitutiven HER2-Rezeptoraktivierung, zu einer unkontrollierten 


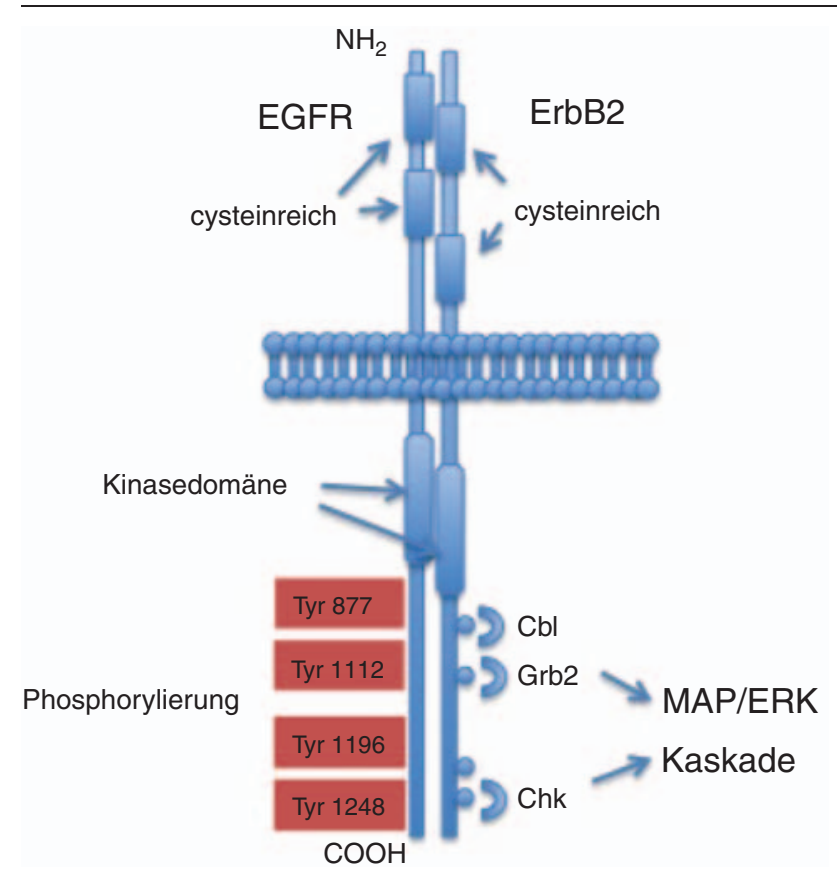

Abb. 1: EGFR/HER2 Heterodimer-assoziierte Rezeptorphosphorylierung

Zellproliferation und zu einem prognostisch besonders ungünstigen Phänotyp. Die Entwicklung einer HER2spezifischen Antikörpertherapie mit Trastuzumab hat HER2-überexprimierende Mammakarzinome inzwischen aber auch zu einem selektiv angreifbaren Ziel gemacht.

Die einzelnen Mitglieder der HER-Familie sind miteinander durch ein komplexes, im Detail noch nicht komplett aufgeklärtes Netzwerk aus Signaltransduk- tionselementen verbunden, und die selektive Hemmung eines Rezeptortyps kann aufgrund von Redundanzen in der Signaltransduktion durch vermehrte Aktivierung anderer Rezeptoren kompensiert werden [1]. Die beiden entscheidenden Schenkel der HER-Signalweitergabe sind der Ras/Raf-Schenkel, über den Apoptose reguliert wird, und der PI3K/AKT-Schenkel, der zelluläre Proliferation steuert (Abb. 2).

HER2 ist der wichtigste Heterodimerisierungspartner von EGFR. Es verstärkt die EGFR-mediierte Signaltransduktionskaskade durch Erhöhung der Bindungsaffinität und durch eine Verlängerung der Rezeptorhalbwertszeit [2, 3]. Umgekehrt scheint auch EGFR-Aktivierung einen Einfluss auf die HER2-Aktivität zu haben: Seit längerem ist bekannt, dass die Aktivierung von EGFR durch seinen Liganden EGF zu einer Heterodimerisierung mit HER2 führt, was wiederum eine HER2-Rezeptoraktivierung bewirkt. Eine Hemmung von EGFR mittels selektiver TKIs führt in der Tat zu einer Wachstumshemmung von HER2-überexprimierenden Brustkrebszelllinien in vitro und in vivo [4]. Diese Untersuchungen lassen den Schluss zu, dass eine kombinierte Hemmung von HER2 und EGFR wirksamer sein dürfte als die Hemmung nur eines der beiden Rezeptoren.

Hemmung der HER-Rezeptorfamilie durch Antikörper Im Prinzip können sowohl an der Zelloberfläche angreifende Antikörper als auch kleine Moleküle

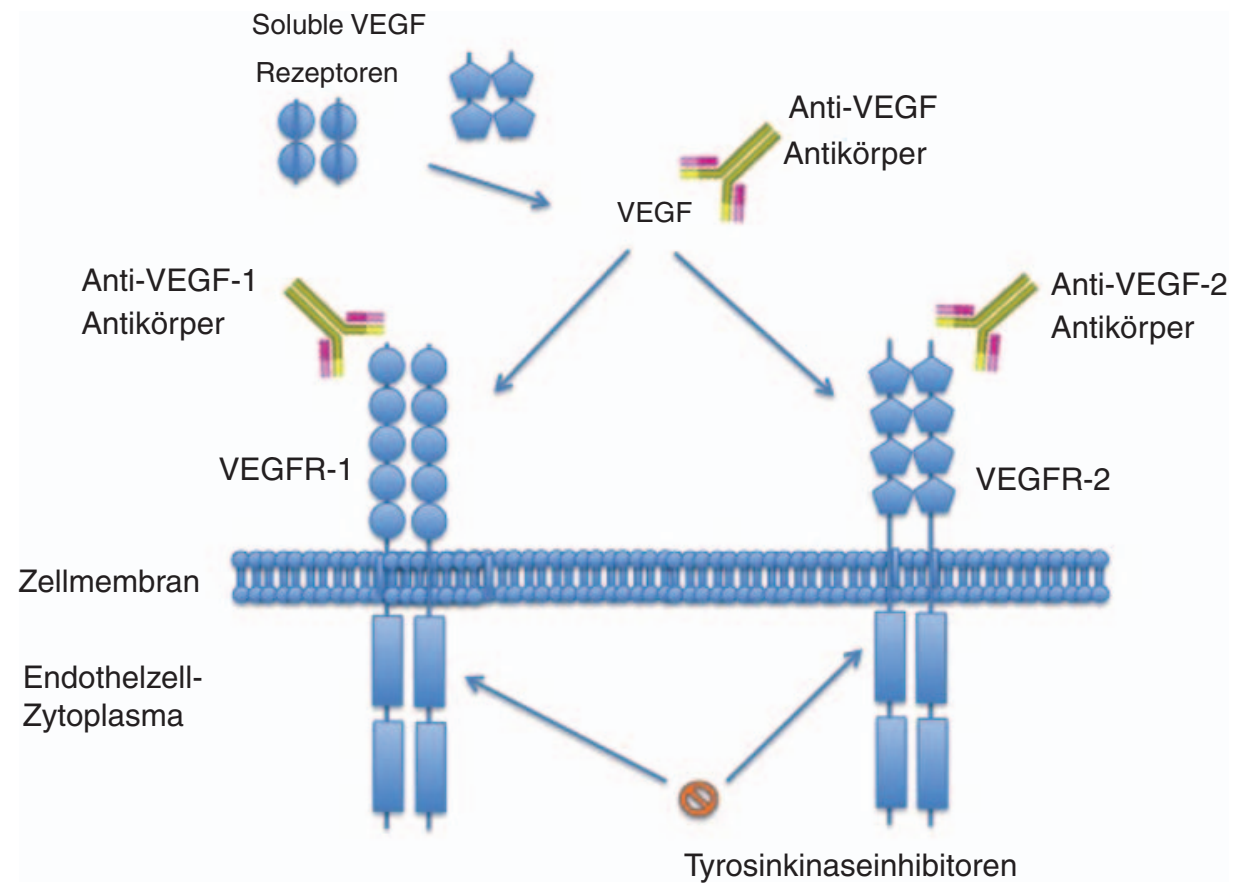

Abb. 2: Strategien zur Hemmung der VEGF-Signaltransduktion: Neutralisierung von VEGF durch Anti-VEGF-Antikörper; Hemmung der Rezeptoraktivierung durch Anti-VEGFR-Antikörper; Hemmung der rezeptorspezifischen Tyrosinkinasephosphorylierung durch Inhibitoren (TKIs) 
(„small molecules“) die intrazelluläre Tyrosinkinasen blockieren und eine Rezeptor-mediierte Signaltransduktion hemmen. Antikörper wirken gewöhnlich als Dimerisierungshemmer und verhindern so die Rezeptorauto-und-transphosphorylierung, die für eine Signalweitergabe essentiell sind [5].

\section{Trastuzumab}

Trastuzumab (Herceptin ${ }^{\circledR}$ ) ist ein humanisierter monoklonaler Antikörper, der die extrazelluläre Domäne des HER2-Rezeptors selektiv bindet und eine Rezeptoraktivierung verhindert. Die klinische Bedeutung von Trastuzumab beim HER2 überexprimierenden Mammakarzinom ist in einer Reihe von Review-Artikel bereits ausführlich beschrieben worden [6].

\section{Pertuzumab}

Pertuzumab ist ein weiterer humanisierter Antikörper, der an die extrazelluläre Domäne von HER2 bindet. Im Gegensatz zu Trastuzumab bindet er aber nicht an die Rezeptordomäne IV, sondern an die Dimerisierungsdomäne II, und hemmt so die Dimerisierung mit HER2 mit EGFR und HER3, und in der Folge die Ligandabhängige Aktivierung der Heterodimer-Komplexe [7]. Tierexperimentelle Studien mit HER2-überexprimierenden Xenografttumoren deuten darauf hin, dass die Kombination von Pertuzumab mit Trastuzumab synergistische Effekte aufweist [8]. In der Tat konnte in einer Phase-II-Studie kürzlich gezeigt werden, dass in 66 Patientinnen mit HER2-überexprimierenden, unter Trastuzumab progredienten Karzinomen die neuerliche Gabe von Trastuzumab, nun in Kombination mit Pertuzumab, einen klinischen Nutzen von $50 \%$ erreicht [9]. Die überaus ermutigenden Ergebnisse dieser Studie haben inzwischen zu einer prospektiv randomisierten 2-armigen Phase-IIIRegistrierungsstudie (Clinical Evaluation of Pertuzumab and Trastuzumab, „CLEOPATRA“) geführt, in der HER2-überexprimierende fortgeschrittene Mammakarzinome mit Trastuzumab und Docetaxel mit oder ohne Pertuzumab behandelt werden [10].

\section{Inhibition von HER1 und HER2}

durch Tyrosinkinaseinhibitoren (TKI)

Im Gegensatz zu Antikörpern müssen TKIs in die Zelle eindringen, um ihre Wirkung zu entfalten. Sie sind daher klein (daher auch die Bezeichnung „small molecules"), und hemmen je nach Struktur mehrere Phosphorylierungsstellen. Dadurch lässt sich die Aktivierung gleich mehrerer Mitglieder der HER-Familie simultan hemmen. Wie bereits ausgeführt, scheint die duale Inhibition von EGFR und HER2 einen synergis- tischen Effekt auf die Wachstumshemmung von HER2-überexprimierenden Tumorzellen zu besitzen. Aber auch aufgrund einer Redundanz von Signaltransduktionsmechanismen, die bei der selektiven Hemmung eines einzelnen Rezeptors über eine Hochregulation von anderen Mitgliedern der HER-Familie zum Auftreten von Resistenzen führt, könnte eine simultane Blockade mehrerer Rezeptoren klinisch vorteilhaft sein.

\section{Lapatinib}

Lapatinib (GW572016, Tyverb ${ }^{\circledR}$ ) ist ein orales 4-Aminoquinazolinederivat, das die intrazelluläre Tyrosinkinasedomäne von EGFR und HER2 reversibel bindet und so inhibiert. Im Gegensatz zu Erlotinib und Genfitinib, die den EGFR in seiner aktiven Konformation binden, greift Lapatinib am inaktiven Rezeptor an. Diese Eigenschaft erklärt, warum Lapatinib eine langsamere Dissoziationsrate und damit einen deutlich länger anhaltenden Effekt aufweist als die beiden vergleichbaren TKIs [11]. Lapatinib ist in Kombination mit Capecitabine seit 2008 in der Therapie des fortgeschrittenen HER2-überexprimierten Mammakarzinoms in der EU zugelassen [12].

\section{Angiogenesehemmung}

\section{Bedeutung der Angiogenese beim Mammakarzinom}

Als Angioneogenese bezeichnet man die Entwicklung von neuen Blutgefäßen aus bereits bestehenden Strukturen. Dieser Prozess läuft normalerweise in mehreren koordinierten Schritten ab und ist beim gesunden Erwachsenen essentiell auf Wundheilung und Schwangerschafts-assoziierte Veränderungen beschränkt. Vor mehr als 35 Jahren hat Judah Folkman erstmals die Bedeutung der Angiogenese auch bei der Entstehung und Ausbreitung von bösartigen Tumoren postuliert und die Hemmung der Angiogenese als mögliche therapeutische Option beschrieben. In der Tat benötigt ein Tumor ab einer kritischen Größe Blutgefäße, um eine ausreichende Oxygenierung und Nährstoffzufuhr für die einzelnen Tumorzellen zu ermöglichen. Hypoxieassoziierte Faktoren wie der Hypoxia-inducible Factor (HIF) werden von Tumorzellen als Folge eines erniedrigten Sauerstoffpartialdrucks freigesetzt und induzieren die Expression von Vascular-Epithelial Growth Factors („VEGFs“). Allerdings scheinen bestimmte Tumorzellen auch bei normalem Sauerstoffpartialdruck bestimmte VEGF-Isoformen zu produzieren. VEGF A-D, Transforming Growth Factor (TGF) alpha, sowie placental growth factors binden ihrerseits mit 
unterschiedlicher Affinität an Endothelzell-ständige Tyrosinkinaserezeptoren, wie beispielsweise VEGF-R 1-3, und induzieren die Endothelzellproliferation und eine erhöhte vaskulare Permeabilität [13]. Die Expression von VEGFs kann durch andere transmembranäre Tyrosinkinaserezeptoren wie beispielsweise EGFR und HER2 hochreguliert werden. Dieser Umstand hat insbesondere bei HER2-überexprimierenden Tumoren potentiell große klinische Relevanz, da die Kombination einer antiangiogenetischen und einer Anti-HER2-Therapie in derartigen Karzinomen einen potentiell synergistischen Effekt aufweist [14].

\section{Bevacizumab}

Bevacizumab (Avastin ${ }^{\circledR}$ ) ist ein rekombinanter VEGFAntikörper, der praktisch alle VEGF-A Isoformen erkennen und in der Folge eine Bindung an den Rezeptor verhindern kann. Bevacizumab ist der bis heute mit Abstand bestuntersuchte Angiogeneseinhibitor und aufgrund seiner überzeugenden klinischen Wirksamkeit seit 2007 in der EU in Kombination mit Taxanen zur Therapie des metastasierten Mammakarzinoms zugelassen.

\section{VEGF-Hemmung durch Tyrosinkinase- Inhibition}

\section{Sorafenib}

Im Gegensatz zu Bevacizumab kann das relativ kleine Bis-aryl Harnstoffderivat Sorafenib (BAY-43-9006) problemlos durch die Zellmembran translozieren und an den intrazellulären Tyrosinkinasen unterschiedlicher Rezeptoren binden. Das oral verfügbare Molekül hemmt die TK-mediierte Signaltransduktion von VEGF-R 2 und 3, den Platelet-Derived Growth Factor Receptor (PDGF-R), von Raf-1 und sowohl der WildtypForm als auch der mutierten (V600E) Form von B-Raf. Seine antineoplastische Aktivität scheint damit sowohl auf einem antiangiogenetischen als auch auf einem direkten antiproliferativen und einem pro-apoptotischen Effekt zu beruhen. Das klinische Potential dieser Substanz wurde in einer Phase-II-Studie bei 54 Patientinnen mit fortgeschrittenem Brustkrebs evaluiert, in der es zwar nur bei einer einzigen Patientin zu einer partiellen Remission kam, allerdings bei $37 \%$ $\mathrm{zu}$ einer Tumorstabilisation. Die Tatsache, dass $22 \%$ der Frauen noch nach 4 Monaten, und $11 \%$ noch nach 6 Monaten eine Stabilisation aufwiesen, könnte darauf hindeuten, dass Multikinaseinhibitoren wie Sorafenib wohl eher die Tumorkinetik derart beeinflussen, dass sie ein Fortschreiten der Krebserkrankung verhindern, als dass sie einen direkten zytoreduktiven Effekt aufweisen.

\section{Sunitinib $\left(\right.$ Sutent $\left.^{\circledR}\right)$}

Sunitinibmalat (SU11248, Sutent ${ }^{\circledR}$ ) ist ein oraler TKI, der ebenfalls mehrere intrazellulären Angriffspunkte besitzt. Das kleine Molekül hemmt selektiv die Signaltransduktion der VEGF-Rezeptoren Typ 1 und 2, PDGF$\mathrm{R}$ a und $\mathrm{b}$, von c-kit und von RET und greift, ähnlich wie Sorafenib, sowohl in die Zellproliferation als auch in die Angiogenese ein [15]. Je nach Expressionsstatus und pathophysiologischer Bedeutung dieser Rezeptoren bei bestimmten Tumorentitäten ist die Substanz mehr oder weniger effektiv. Da insbesondere PDGF-R beim Mammakarzinom eine wichtige Rolle bei der parakrinen Steuerung der Tumorzellproliferation $\mathrm{zu}$ besitzen scheint, wurde Sunitinib bereits in einer kleinen Phase-II-Studie in 64 Frauen mit fortgeschrittenem Mammakarzinom untersucht, die zuvor sowohl mit Anthrazyklinen als auch mit Taxanen erfolglos behandelt worden waren. Die tägliche Gabe von $50 \mathrm{mg}$ durch 4 Wochen innerhalb eines 6-wöchentlichen Zyklus führte bei immerhin $11 \%$ zu einer partiellen Remission, während weitere $5 \%$ der so behandelten Frauen eine Tumorstabilisation für die Dauer von mehr als 6 Monaten aufwiesen [16]. Auch Sunitinib scheint in Kombination mit Trastuzumab synergistische antitumorale Eigenschaften zu besitzen. In einer Phase-II-Studie bei Frauen mit HER2-überexprimierendem, Trastuzumabnaivem Mammakarzinom, konnte durch eine Kombination von Sunitinib mit Trastuzumab und Docetaxel in der Erstlinientherapie ein objektives Ansprechen von beinahe $78 \%$ erzielt werden. Die Ansprechrate der Sunitinib-Kombination liegt damit deutlich über jener, die üblicherweise in diesem Setting durch die Gabe von Trastuzmab und Docetaxel erreicht werden kann [17].

\section{Interessenskonflikt}

Es besteht kein Interessenskonflikt.

\section{Literatur}

[1] Yarden Y, Sliwkowsky MX. Untangling the ErbB signaling network. Nat Rev Cell Mol Biol, 2: 127-137, 2001.

[2] Karunagaran D, Tzahar E, Beerli RR, et al. ErbB-2 is a common auxiliary subunit of NDF and EGF receptors: implications for breast cancer. EMBO J, 15: 254-264, 1996.

[3] Worthylake R, Opresko LK, Wiley HS, et al. ErbB-2 amplification inhibits down-regulation and induces constitutive activation of both ErbB-2 and epidermal growth factor receptors. J Biol Chem, 274: 8865-8874, 1999.

[4] Moulder SL, Yakes FM, Muthuswamy SK, et al. Epidermal growth factor receptor (HER1) tyrosine kinase inhibitor ZD1839 (Iressa) 
inhibits HER2/neu (erbB2)-overexpressing breast cancer cells in vitro and in vivo. Cancer Res, 61: 8887-8895, 2001.

[5] Murphy CG, Fornier M. HER2-positive breast cancer: beyond trastuzumab. Oncology (Williston Park), 24(5): 410-415, 2010.

[6] Tkaczuk KH. Review of the contemporary cytotoxic and biologic combinations available for the treatment of metastatic breast cancer. Clin Ther, 31(Pt 2): 2273-2289, 2009. Review.

[7] Franklin MC, Carey KD, Vajdos FF, et al. Insights into ErbB signaling from the structure of the ErbB2-pertuzumab complex. Cancer Cell, 5: 317-328, 2004

[8] Nahta R, Hung MC, Esteva F. The HER-targeting antibodies trastubzmab and pertuzumab synergistically inhibit the survival of breast cancer cells. Cancer Res, 64: 2343-2346, 2004.

[9] Gelmon KA, Fumoleau P, Verma S, et al. Results of a phase II trial of trastuzumab and pertuzumab in patients with Her2-positive metastatic breast cancer who had progressed during trastuzumab therapy. J Clin Oncol, 26(Suppl 15S): 47s, 2008.

[10] Baselga J, Gelmon KA, Verma S, Wardley A, Conte P, Miles D, Bianchi G, Cortes J, McNally VA, Ross GA, Fumoleau P, Gianni L. Phase II trial of pertuzumab and trastuzumab in patients with human epidermal growth factor receptor 2-positive metastatic breast cancer that progressed during prior trastuzumab therapy. J Clin Oncol, 28(7): 1138-1144, 2010. Epub 2010 Feb 1.

[11] Wood ER, Truesdale AT, McDonald OB, et al. A unique structure for epidermal growth factor receptor bound to GW572016
(Lapatinib): relationships among protein conformation, inhibitor off-rate, and receptor activity in tumor cells. Cancer Res, 64: 6652-6659, 2004.

[12] Untch M. Targeted therapy for early and locally advanced breast cancer. Breast Care (Basel), 5(3): 144-152, 2010. Epub 2010 Jun 16

[13] Neufeld G, Cohen T, Gengrinovitch S, et al. Vascular endothelial growth factor (VEGF) and its receptors. FASEB J, 13: 9-22, 1999.

[14] Pegram MD, Konecny GE, O'Callaghan C, et al. Rational combinations of trastuzumab with chemotherapeutic drugs used in the treatment of breast cancer. J Natl Cancer Inst, 96: 739-749, 2004.

[15] Polyzos A. Activity of SU11248, a multitargeted inhibitor of vascular endothelial growth factor receptor and platelet-derived growth factor receptor, in patients with metastatic renal cell carcinoma and various other solid tumors. J Steroid Biochem Mol Biol, 108: 261-266, 2008.

[16] Miller KD, Burstein HJ, Elias AD, et al. Phase II study of SU11248, a multitargeted receptor tyrosine kinase inhibitor (TKI) in patients with previously treated metastatic breast cancer (MBC). In: Proceedings of the American Society of Clinical Oncology, Annual Meeting, Orlando, FL, 2005. (Abstract 563)

[17] Bachelot T, Lluch A, Gutierrez M, et al. Activity and Safety of Sunitinib in Combination with Trastuzumab for the Treatment of Metastatic Breast cancer: Initial Results from a Phase II Study. 31. SABCS, San Antonio, 2008. (Abstract 4115) 